\title{
Mapping of Two Dimensional Doping Areas in CMOS Device by Using Transport of Intensity Equation
}

\author{
Wen-Kuo Hsieh, Fu-Rong Chen, Yi-Ming Chen, Hsiao-Wen Hsu and Ji-Jung Kai
}

Center for Electron Microscopy, Department of Engineering and System Science, National

Tsing-Hua University, Hsinchu, Taiwan. 30043, R.O.C.

Understanding the dopant diffusion in a device is one of the major challenges in advance ULSI semiconductor technology of nowadays. Recently, applications of electron holography in transmission electron microscopy to map 2D dopant distribution in the phase image have shown considerable promise [1]. However, the preparation of samples for analysis is a crucial aspect of the application of electron holography to semiconductor materials.

The non-interferometric method, involving the so-called Transport of Intensity Equation (TIE) [2], have potentially a practical method for quantifying $2 \mathrm{D} p-n$ junctions as used in electron holography. The phase retrieval procedure with TIE has been successfully applied in different fields [3-5]. Here, we propose that TIE can be solved with a two-step de-convolution process using the maximum entropy method (MEM) is a sensitive phase trivial method that potentially provides comparable results as those obtained using electron holography.

The p-n junctions for this investigation were made on a p-type Si wafer by implanting arsenic ions at $2 \mathrm{KeV}$ to a dose of $9 \times 10^{14} \mathrm{~cm}^{-2}$, then annealing at $1075{ }^{\circ} \mathrm{C}$. Thin cross section of the device suitable for TEM observation was prepared by regular procedure and experimental images were obtained using a JEOL 2010F FEGTEM at 200kV. Figure 1(a)-(e) shows a series of cross-sectional TEM images of an $n$-MOS (As doped) transistor. The images were separated by a step size of 1510.4 $\mathrm{nm}$. The Figure 2 presents the structure of n-MOS which demonstrated in the paper. The compositions and expected doping regions also indicated in the schematic. Figure 3 (a)-(b) show the reconstructed phase image by TIE/MEM method. The main characteristics of the n-MOS device were reconstructed as shown in phase image. The source and drain areas of the device are clearly visible by the increase in contrast. Because of the phase shift of the electron wave is proportional to inner potential of the material which electron wave passes through. Therefore, the whiter contrast suggests that the potential is higher than the dark regions. It's reasonable that the potential in the doped n-type (As doped) region is higher than the p-type (B doped) silicon substrate.

The maximum entropy de-convolution method (MEM) is employed to solve the transport of intensity equation (TIE) for phase retrieval problems. The results establish that TIE/MEM can become an efficient and potentially a practical method for quantifying $2 \mathrm{D} p-n$ junctions as used in electron holography.

\section{Reference}

[1] W. D Rau, P. Schwander, F. H. Baumann, W. Höppner, and A. Ourmazd, Phys. Rev. Lett. 82 (1999) 2614

[2] M. R. Teague. J. Opt. Soc. Am. 73 (1983) 1434

[3] T. E. Gureyev, and K. A. Nugent, Opt. Commun.133 (1997) 339

[4] W. K. Hsieh, et al. Ultramicroscopy 98 (2004) 99-114

[5] B. E, Allman, et al. , Nature 408 (2000) 158 

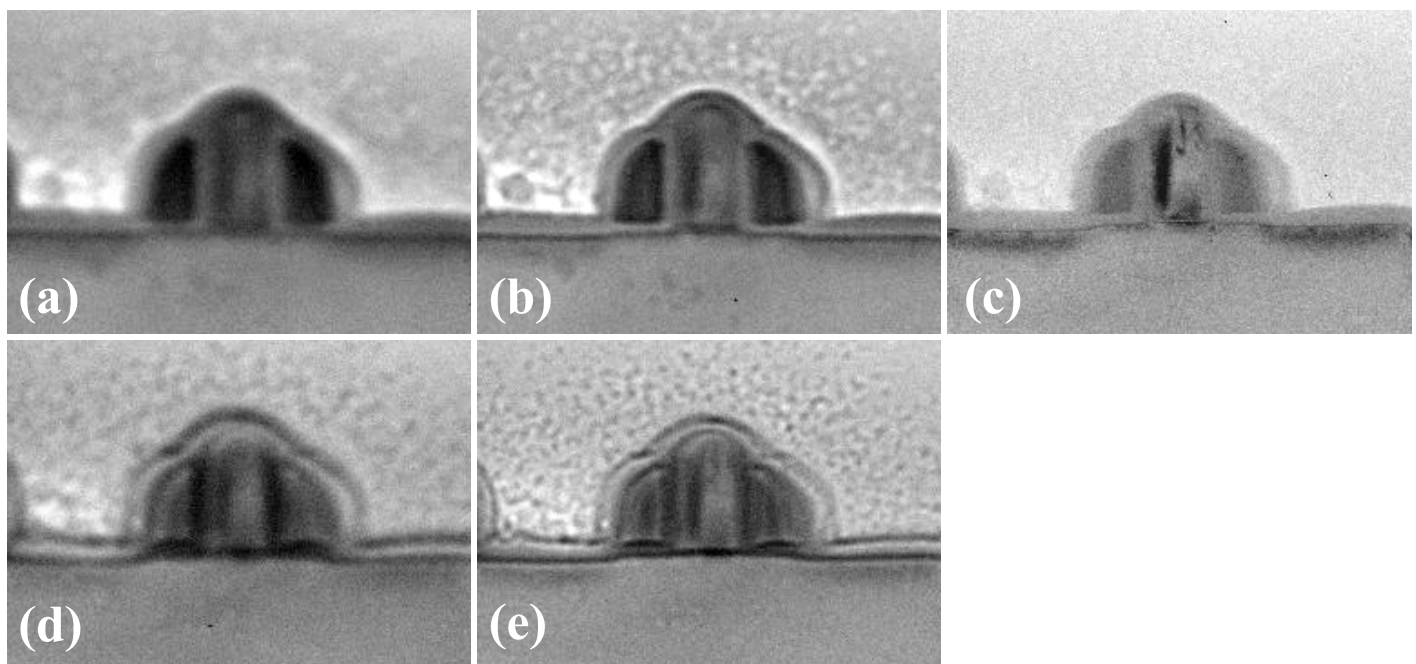

Figure 1(a)-(e)

TEM images of $n$-MOS (As doped) transistor with $90 \mathrm{~nm}$ channel length

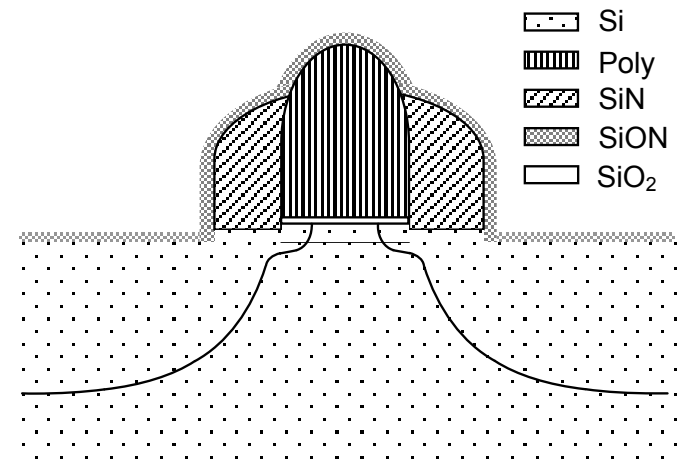

Figure 2

The structure of n-MOS device demonstrated in the experiment.
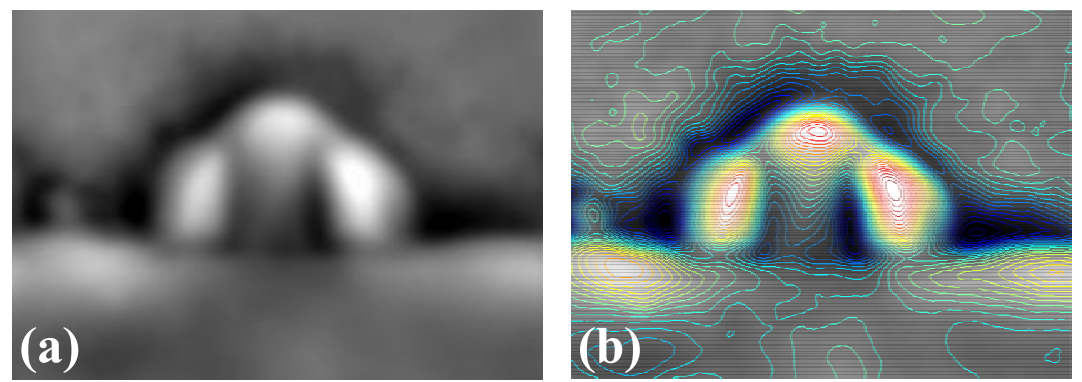

Figure 3

(a) Reconstructed phase image by using TIE/MEM method

(b) Phase image presents in equal phase contour map. 